\title{
First transitions in circular Couette flow with axial stratification
}

\author{
Bach Lien Hua \\ Laboratoire de Physique des Oceans, ERS 130 CNRS, IFREMER, 29280 Plouzané, France \\ Sylvie Le Gentil \\ Laboratoire de Physique des Oceans, ERS 130 CNRS, IFREMER, 29280 Plouzané, France \\ Paolo Orlandi \\ Dipartimento di Meccanica e Aeronautica Università di Roma “La Sapienza," Via Eudossiana 18, 00184, \\ Roma, Italy
}

(Received 28 May 1996; accepted 14 October 1996)

\begin{abstract}
The first flow regimes which have been observed experimentally for a circular Couette flow with a stable, axial stratification in density are investigated through direct numerical simulations of the three-dimensional Navier-Stokes equations for a Boussinesq fluid. The setup of two concentric cylinders has a nondimensional gap width of $\epsilon=(b-a) / a=0.289$; the outer cylinder is fixed and the stratification in density in the axial direction is linear. The main effect of an axial density stratification is to reduce the height of the Taylor vortices and to cause the formation of density layers of small aspect ratio. For large enough Prandtl number, the primary bifurcation from circular Couette flow is found to be axisymmetric and of Hopf-type in the direct numerical simulations. An analytical solution for onset of instability and slightly different boundary conditions from the experimental ones agrees within $0.6 \%$ with numerical simulations at a Prandtl number of 700 . The experimental flow regimes with well-defined density layers are well reproduced by the numerical simulations in the appropriate range of relative Reynolds number $\operatorname{Re} / \operatorname{Re}_{c 1}$, where $\operatorname{Re}_{c 1}$ denotes the critical Reynolds number for the primary bifurcation from circular Couette flow. However, the increase of axial scale with $R e / \operatorname{Re}_{c 1}$ is found to be continuous, whereas it is quantized in the laboratory experiments. Numerical results reveal that the first two transitions between the flow regimes are primarily due to the temporal behavior of the axially symmetric part of the flow. Onset of nonaxisymmetric motions appears at the same $\operatorname{Re} / \mathrm{Re}_{c 1} \approx 1.18$ as in the homogeneous fluid case at the same $\eta=a / b$. Stratification precludes large axial displacements and the azimuthal modes patterns have a quite distinct appearance from the homogeneous wavy modes. At large enough Re, a destabilization of the jet-like outflow between pairs of vortices causes the suppression of the density front which is located at the same axial height. This nonaxisymmetric flow regime presents common features with the wavy outflow boundary (WOB) pattern, which is commonly observed in the homogeneous Couette-Taylor case. (C) 1997 American Institute of Physics.
\end{abstract} [S1070-6631(97)00702-2]

\section{INTRODUCTION}

Our purpose is to investigate through direct numerical simulations some of the flow regimes which have been observed in the laboratory experiments of Boubnov et al. ${ }^{1}$ (denoted by BA, hereafter). We use the three-dimensional Navier-Stokes code which has been developed by Verzicco and Orlandi, ${ }^{2}$ with an additional equation for density. The experimental setup in BA (Fig. 1) concerns the flow between two concentric cylinders, the outer of which is fixed and the inner which rotates at an angular velocity $\Omega$, for a fluid with a stable linear density stratification $N$ in the axial direction. Their apparatus dimensions $\operatorname{are}^{3} a=4.01 \mathrm{~cm}, b=5.15 \mathrm{~cm}$ ( $\eta=a / b=0.775$ ), and corresponds to a nondimensional gap width $\epsilon=(b-a) / a=0.289$, for which the critical inner cylinder speed for onset of instability is $\Omega \approx 0.194 \mathrm{~s}^{-1}$ for the homogeneous fluid case. ${ }^{4}$ The values of buoyancy frequency $N$ which are used by BA lie between 0.35 and $1.78 \mathrm{~s}^{-1}$. These experiments have been devised in order to clarify the role of an axial stratification in the Taylor-Couette problem, which has become a paradigm for understanding problems of hydrodynamic stability and pattern formation. ${ }^{5}$
Our original motivation comes from geophysical observations, especially in the atmosphere and ocean, where centrifugal instability (also called inertial instability in geophysical problems) always occurs within stratified flows. Both the atmosphere ${ }^{6,7}$ and ocean, ${ }^{8}$ equatorial regions correspond to the location of the maximum angular momentum of the fluid and small deviations in the symmetries of the flow are likely to trigger centrifugal instabilities. Symmetry breaking is immediately induced by the existence of a nonzero latitudinal shear at the equator and low-latitude regions are thus privileged locations for the occurrence of inertial instability. There is a close analogy between the equatorial barotropic inertial instability problem, where a depth-independent shear flow in a stratified fluid leads to secondary cellular motions, and the cylindrical Taylor-Couette problem with a stable axial stratification in density. ${ }^{8}$ The symmetries of the two problems are such that the analogy is valid for both linear instability and nonlinear flow regimes.

The laboratory experiments reveal that an axial density stratification has an overall stabilizing effect on the flow, with a clear dependence of the critical Reynolds number on buoyancy frequency $N$, where the Reynolds number is de- 


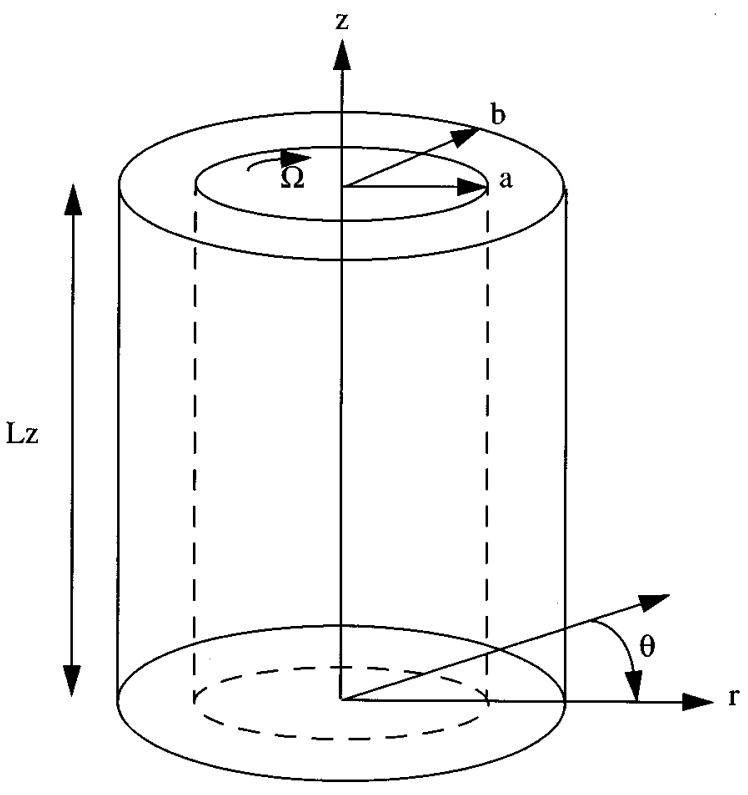

FIG. 1. Coordinate system and the integration domain. The fluid is stably stratified in density in the axial direction.

fined as $\operatorname{Re}=\Omega a d / \nu, \nu$ is the kinematic viscosity of the fluid and $d=b-a$ is the gap width between the two cylinders. This dependence is particularly sensitive near onset of instability, where a stable axial density stratification plays the same stabilizing role as rotation in the thermal convection problem. ${ }^{9-11}$ In both problems, the instability is due to two perpendicular body forces the centrifugal/Coriolis and buoyancy forces, and their destabilizing/stabilizing roles are interverted between the two situations. The selected, most amplified, vertical wavelength at onset of instability is about half the gap width for the experimental geometry which is considered by BA, and for the range of buoyancy frequency values which have been used. The resulting vortex motion causes some mixing and this leads to layer formation, well visible on shadowgraph images. As expected intuitively, the effect of stratification is to delay the occurrence of the secondary rolls and to reduce their height, since stratification tends to favor horizontal motions and to inhibit vertical motions.

The flow regimes which have been observed by BA are reproduced in Fig. 2 as a function of the dimensionless Taylor and Grashof numbers,

$$
T=\frac{4 \eta^{2}}{1-\eta^{2}} \operatorname{Re}^{2}, \quad G=\frac{N^{2} d^{4}}{\nu^{2}} .
$$

To emphasize the dominant role of stratification in Fig. 2, the first regime of vertically reduced vortex size, and density layers of height $h$ such that $n_{l}=h / d \approx \frac{1}{2}$, has been referred to as the $S$-regime by BA. Stratification appears to inhibit the usual azimuthal wavy vortex flow patterns which are observed in the case of homogeneous fluid, but a clear azimuthal dependence of the flow has been noted by BA (their Fig. 4 corresponds to $\operatorname{Re} / \operatorname{Re}_{c 1}=1.3$, where $\operatorname{Re}_{c 1}$ is the critical Reynolds for onset of the $S$-regime). (The sequence of

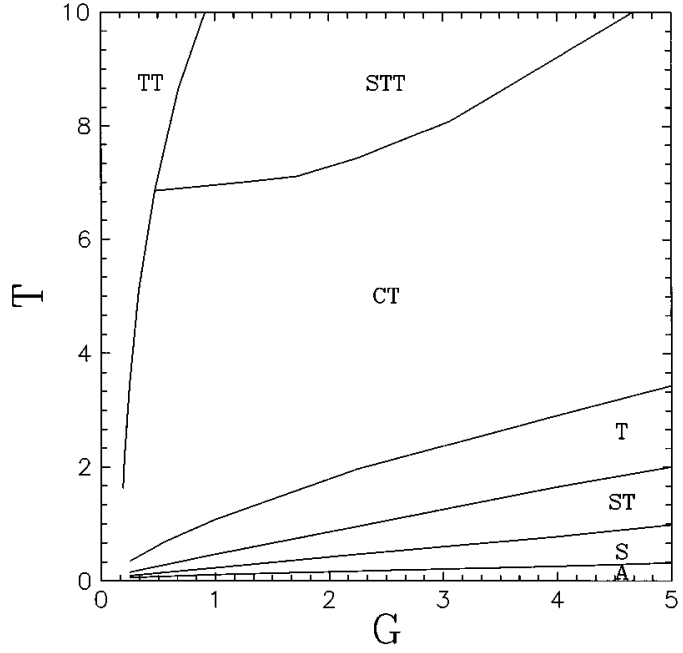

FIG. 2. Experimentally determined flow regimes for stratified circular Couette flow (reproduced from $\mathrm{BA}^{1}$ ) as a function of the Grashof and Taylor numbers $G$ and $T$. Units along the abcissa and ordinate are, respectively, $10^{4}$ and $10^{5}: A$, stable azimuthal flow; $S$, stratification dominated flow with density layer height $n_{l} \approx 1 / 2 ; S T$ transition between $n_{l} \approx 1 / 2$ and $n_{l}=1 ; T$, Taylor vortices with $n_{l}=1 ; C T$, compacted Taylor vortices with $n_{l}=2$; $S T T$, turbulent motion with $n_{l}=2$ and observable layers after stop, $T T$, turbulent mixing without layers after stop. The lines drawn in the figure indicate the approximate boundaries between the different regimes.

transitions between the various flow regimes in Fig. 2 are denoted by subscript $\operatorname{Re}_{c i}, i=1, \ldots 4$.) At larger Reynolds number, the role of stratification decreases and the most amplified axial wavelength increases. After a transition regime $S T$, where several scales are observed to coexist, the next clear flow regime, which is labeled $T$, corresponds to density layers height $h$, which is equal to the gap width $\left(n_{l} \approx 1\right)$. The secondary vortices are observed experimentally to have in that case the same height as that of the classical Taylor vortices in the homogeneous fluid case. At still larger Reynolds number, the Taylor vortices interact by pairs and density layers of twice the gap width appear to be predominant $\left(n_{l} \approx 2\right)$. This regime of "compacted Taylor vortices" has been labelled $C T$. Finally, by further increasing the Reynolds number, turbulent motions appear with superimposed Taylor vortices as in the homogeneous fluid case.

In this paper, we focus our attention on the numerical simulations of the $S, T$ and $C T$ regimes. We want in particular to clarify the role of the Prandtl number (Sec. III) (or rather the Schmidt number, since the linear stratification which is used in BA is accomplished by using a salt solution). The aim of Sec. IV is to identify which parameters control the growth of axial scales which is observed for increasing Re in the sequence of flow regimes. We have restricted our simulations to a single value of the buoyancy frequency, $N=1.04 \mathrm{~s}^{-1}$, which corresponds to one of the experimental values which have been used by BA. The final part of this study concerns the azimuthal dependence of the flow (Sec. V) in the $S$ and $C T$ regime. The Appendix details the analytical solution for onset of instability in the case of slightly different boundary conditions from those of the laboratory experiments. Such solution, which is analogous to 
free-free boundary conditions in the rotating thermal convection problem, is valid for a finite gap width, and has been used to validate the numerical code in its stratified version.

\section{FORMULATION OF THE PROBLEM}

The Navier-Stokes equations for a Boussinesq stratified fluid are used in nondimensional form. The numerical code has been developed by Verzicco and Orlandi ${ }^{2}$ for the variables $\left(q_{\theta}=v_{\theta}, q_{r}=r v_{r}, q_{z}=v_{z}\right)$, where $\left(v_{\theta}, v_{r}, v_{z}\right)$ are the velocity components in cylindrical coordinates. An additional equation for the perturbation density $\rho^{\prime}$ has been added.

The momentum and density equations, when written in conservative form, are given by

$$
\begin{aligned}
& \frac{D q_{\theta}}{D t}=-\frac{1}{r} \frac{\partial p}{\partial \theta}+\frac{1}{\operatorname{Re}}\left[\frac{1}{r}\left(\frac{\partial}{\partial r} r \frac{\partial q_{\theta}}{\partial r}\right)-\frac{q_{\theta}}{r^{2}}+\frac{1}{r^{2}} \frac{\partial^{2} q_{\theta}}{\partial \theta^{2}}+\frac{\partial^{2} q_{\theta}}{\partial z^{2}}+\frac{2}{r^{3}} \frac{\partial q_{r}}{\partial \theta}\right], \\
& \frac{D q_{r}}{D t}=-r \frac{\partial p}{\partial r}+\frac{1}{\operatorname{Re}}\left[r \frac{\partial}{\partial r}\left(\frac{1}{r} \frac{\partial q_{r}}{\partial r}\right)+\frac{1}{r^{2}} \frac{\partial^{2} q_{r}}{\partial \theta^{2}}+\frac{\partial^{2} q_{r}}{\partial z^{2}}-\frac{2}{r} \frac{\partial q_{\theta}}{\partial \theta}\right], \\
& \frac{D q_{z}}{D t}=-\frac{\partial p}{\partial z}+\frac{1}{\operatorname{Re}}\left[\frac{1}{r} \frac{\partial}{\partial r}\left(r \frac{\partial q_{z}}{\partial r}\right)+\frac{1}{r^{2}} \frac{\partial^{2} q_{z}}{\partial \theta^{2}}+\frac{\partial^{2} q_{z}}{\partial z^{2}}\right]+\frac{G}{\operatorname{Re}^{2} \rho^{\prime}}, \\
& \frac{D \rho^{\prime}}{D t}=+q_{z}+\frac{1}{\operatorname{RePr}}\left[\frac{1}{r} \frac{\partial}{\partial r}\left(r \frac{\partial \rho^{\prime}}{\partial r}\right)+\frac{1}{r^{2}} \frac{\partial^{2} \rho^{\prime}}{\partial \theta^{2}}+\frac{\partial^{2} \rho^{\prime}}{\partial z^{2}}\right]
\end{aligned}
$$

Notations correspond to

$$
\begin{aligned}
& \frac{D q_{\theta}}{D t}=\frac{\partial q_{\theta}}{\partial t}+\frac{1}{r^{2}} \frac{\partial r q_{\theta} q_{r}}{\partial r}+\frac{1}{r} \frac{\partial^{2} q_{\theta}}{\partial \theta^{2}}+\frac{\partial q_{\theta} q_{z}}{\partial z} \\
& \frac{D q_{r}}{D t}=\frac{\partial q_{r}}{\partial t}+\frac{\partial}{\partial r}\left(\frac{q_{r}^{2}}{r}\right)+\frac{\partial}{\partial \theta}\left(\frac{q_{\theta} q_{r}}{r}\right)+\frac{\partial q_{r} q_{z}}{\partial z}-q_{\theta}^{2} \\
& \frac{D q_{z}}{D t}=\frac{\partial q_{z}}{\partial t}+\frac{1}{r} \frac{\partial q_{r} q_{z}}{\partial r}+\frac{1}{r} \frac{\partial q_{\theta} q_{z}}{\partial \theta}+\frac{\partial q_{z}^{2}}{\partial z} \\
& \frac{D \rho^{\prime}}{D t}=\frac{\partial \rho^{\prime}}{\partial t}+\frac{1}{r} \frac{\partial q_{r} \rho^{\prime}}{\partial r}+\frac{1}{r} \frac{\partial q_{\theta} \rho^{\prime}}{\partial \theta}+\frac{\partial q_{z} \rho^{\prime}}{\partial z}
\end{aligned}
$$

The continuity equation is

$$
\frac{\partial q_{r}}{\partial r}+\frac{\partial q_{\theta}}{\partial \theta}+r \frac{\partial q_{z}}{\partial z}=0
$$

Equations are nondimensionalized by a reference velocity which corresponds to the inner cylinder velocity $\Omega a$; the reference length is the gap width $d$. The buoyancy fluctuation $\rho^{\prime}=g(\overline{\rho(z)}-\rho) /\left(\rho_{0} N^{2} d\right)$ is the nondimensional deviation from the background density field $\overline{\rho(z)}$ $=\rho_{0}\left(1-N^{2} / g z\right)$, where $g$ denotes gravitational acceleration. The Prandtl number is $\operatorname{Pr}=\nu / \kappa$, where $\kappa$ is the density diffusivity and $\nu$ is the viscosity.

The system of equations is integrated by a finitedifference scheme based on a fractional-step method. ${ }^{2}$ An approximate factorization technique is used for the implicit treatment of the viscous terms and the code accuracy has been tested for several flows in cylindrical coordinates. ${ }^{2} \mathrm{~A}$ nonuniform grid is used in the radial direction, with a refinement of the mesh near the solid boundaries. Initial conditions correspond to a Couette profile,

$$
\bar{q}_{\theta}=\frac{\eta}{1-\eta^{2}}\left[\frac{1}{(1-\eta) r}-(1-\eta) r\right],
$$

and small disturbances are introduced on the vertical velocity $q_{z}$ (see next sections). The incompressibility of the perturbed velocity field is used for determining the initial field of $q_{r}$. External parameters which are assigned are the experimental values of $a, b, \Omega, \nu, \kappa, N$, and from these quantities $\operatorname{Pr}, \operatorname{Re}$ and $G$ are evaluated.

Boundary conditions in the experimental setup of BA correspond to no-slip and insulating radial boundaries for density, since stratification is accomplished by using a salt solution,

(i) $q_{\theta}=q_{r}=q_{z}=0, \partial \rho / \partial r=0$.

Such a set of boundary conditions is appropriate for the experimental conditions of BA. Two other sets of boundary conditions have also been studied, namely

(ii) $q_{\theta}=q_{r}=q_{z}=0$ and $\rho=0$ (isothermal boundary),

(iii) $q_{\theta}=q_{r}=0$ and $\partial q_{z} / \partial r=\partial \rho / \partial r=0$.

The last two sets are respectively analogous to the rigid and free boundary conditions in the rotating thermal convection problem (see the Appendix). In the latter case, a straigthforward analytical solution is available which is valid at a finite gap width, as it is the case in BA's experiments.

The approach is based on the assumption of an infinite cylinder and periodicity is assumed in the vertical direction. The nondimensional vertical extension of the domain of integration is $L_{z} / d$. The choice of $L_{z}$ influences the axial scale of the flow through the axial quantization of the solution which is thus implied.

The bulk of the two-dimensional simulations in $(r, z)$ have been performed at $96 \times 96$, while the three-dimensional simulations have been performed at $96 \times 96 \times 96$ in $(\theta, r, z)$.

We first checked the code accuracy by comparing with published results for the homogeneous Taylor-Couette problem. For the experimental value of $\eta=0.775$ of BA, the critical value for a transition from purely azimuthal Couette flow ${ }^{4}$ is $\Omega_{c}=0.194$, and this value has been reproduced within a relative error of $0.5 \%$. For three-dimensional solu- 
tions, the index for checking the code accuracy is the phase speed of wavy vortex flows, such as prognosed by Jones, ${ }^{12}$ who also considered the case of a fixed outer cylinder. His Fig. 4 corresponds to the case $\eta=0.8756$, and for azimuthal wave number $m=1$, we have checked that at $\operatorname{Re}=1.2 \mathrm{Re}_{c}$ the error in propagation speed is also less than $0.5 \%$, for $L_{z} / d=2$.

Finally, for the stratified fluid case, the numerical accuracy has been validated for the set of boundary conditions (iii), for which analytical solutions for the critical values of the Reynolds number, axial wavelength and oscillation period are given in the Appendix. Such analytical solutions are valid for a finite gap width and we find that, at the experimental value of $\operatorname{Pr}=700$, the numerical results lie within $0.6 \%$ of the analytical solutions. This numerical result (which also validates $a$ posteriori the use of such large values of $\operatorname{Pr}$ ) is due to the fact that the boundary layer thickness for $q_{\theta}^{\prime}$ and $\rho^{\prime}$, is determined by viscosity $\nu$, rather than by diffusivity $\kappa$ at onset of instability.

\section{ONSET OF INSTABILITY AND INFLUENCE OF PRANDTL NUMBER}

Veronis ${ }^{11}$ has detailed several examples which illustrate the dynamical analogy which exists between a stratification in density and a stratification in angular momentum, as initially suggested by Lord Rayleigh. ${ }^{13}$ This analogy has been used $^{10}$ to extend to the stratified Couette-Taylor problem many of the results established for the rotating convection problem in the case of free-free boundaries. ${ }^{9}$ One important result which carries over to our problem is that, for large enough values of the Prandtl number, the primary instability should be oscillatory. This is confirmed by our threedimensional numerical simulations which show that at $N=1.04 \mathrm{~s}^{-1}$ and $\mathrm{Pr}=700$, the first transition is of Hopf type, purely axisymmetric, and occurs for a critical inner cylinder rotation speed of $\Omega_{c 1}=0.404 \pm 0.001 \mathrm{~s}^{-1}$, for a value of $\nu=0.0104 \mathrm{~cm}^{2} \mathrm{~s}^{-1}$, which takes into account the change of the kinematic viscosity of the salt solution. The quantitative impact of a large Prandtl number is the modification of the critical values of the Reynolds number and oscillation period at onset of instability.

The laboratory experiments yield for the inner cylinder rotation speed a critical value of $\Omega_{c 1}^{o b s}=0.409 \mathrm{~s}^{-1}$, and the discrepancy between the experiments and numerical simulations is thus of about $1 \%$. The numerical result for the most unstable axial wavelength $2 h$ of the density field is such that $(h / d)_{c}=0.515 \pm 0.005$, while the laboratory experiments correspond to $h / d=0.51$ (see Fig. 3 in $\mathrm{BA}$ and the corrigendum ${ }^{3}$ ). The discrepancy is again of $1 \%$ between the two approaches. The procedure which is used for determining the critical density layers height is to vary the axial height of the domain of integration $L_{z}=2 h$ until the largest growth rate is obtained in the direct numerical simulation. Onset of instability thus corresponds to $L_{z} / d=1.03 \pm 0.01$.

The Hopf-character of the transition has been checked numerically through a dependence of the amplitude of the convective motions on $\left[\left(\operatorname{Re}-\operatorname{Re}_{c 1}\right) / \operatorname{Re}_{c 1}\right]^{1 / 2}$. The oscillation period is found in the numerical experiments to be $\tau_{c 1}=19.25 \mathrm{~s}$ at onset of instability for the boundary condi-

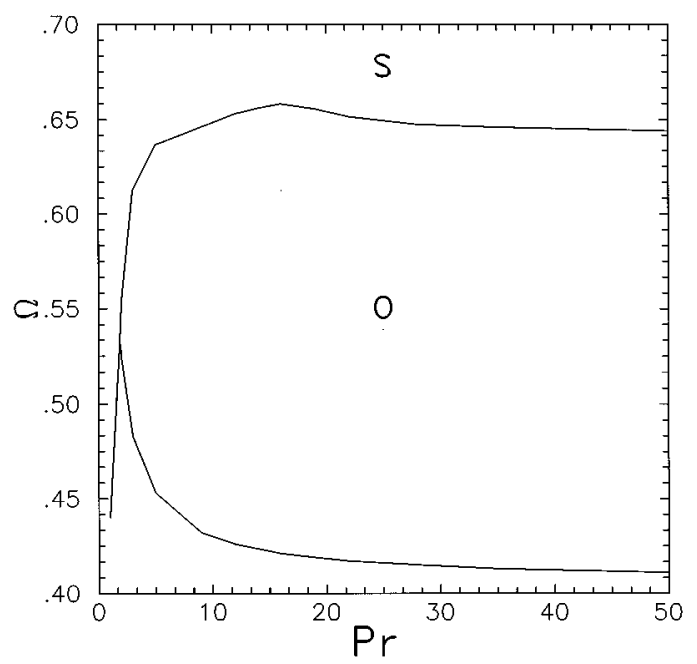

FIG. 3. Numerically determined stability boundary for the critical rotation rate $\Omega$ as a function of Prandtl number, for a buoyancy frequency $N=1.04$ s and $L_{z} / d=1$. Symbols: O, oscillatory regime; S, stationary flow regime.

tions (i) which are the relevant ones for the laboratory experiments. Other numerical simulations using either set (ii) or (iii) reveal a weak dependence of the solutions on the nature of the boundary conditions at onset of instability (see the Appendix). Moreover, the above value of $\tau_{c 1}$ lies within $3 \%$ of the analytical expression

$$
\tau_{c}=\sqrt{2\left(1+\left(\frac{d}{h}\right)^{2}\right)} \frac{2 \pi}{N}
$$

which is established in the Appendix for set of conditions (iii) and corresponds to $\tau_{c}=18.66 \mathrm{~s}$ for $d / h=0.515$.

We note that the primary transition is purely axisymmetric in our three-dimensional simulations, exactly as in the homogeneous fluid problem for a fixed outer cylinder. The numerical simulations reveal furthermore that an azimuthal dependence of the secondary flow only appears at $\operatorname{Re} / \operatorname{Re}_{c} \geqslant 1.18 \pm 0.01$ (see Sec. V). This first flow regime corresponds to the regime which has been labelled $S$ by BA (Fig. 2), but their statement that the flow regime $S$ is nonaxisymmetric is valid only for $\operatorname{Re} / \operatorname{Re}_{c} \geqslant 1.18$. (BA only discuss the case $\operatorname{Re} / \operatorname{Re}_{c}=1.3$ in their Fig. 4.)

We have studied the role of Pr on the temporal dependence of the initial flow regime through axisymmetric simulations at a fixed axial domain height of $L_{z} / d=1$, and the results are given in Fig. 3. The two branches in Fig. 3 delimit the stability boundary as a function of $\Omega$ and $\operatorname{Pr}$, for a transition, respectively, to a stationary flow regime (upper branch) and an oscillatory one (lower branch). As expected from the results of Thorpe, ${ }^{10}$ at low values of the Prandtl number, $\operatorname{Pr} \leqslant 1.87$, the primary transition corresponds to a stationary flow regime, while for $\operatorname{Pr} \geqslant 1.87$, the flow regime is oscillatory. BA's flow regime $S$ corresponds to the latter case. Our numerical result differs however quantitatively from Thorpe's prediction that $\operatorname{Pr}=1.43$, for the limiting value of Prandtl number between the stationary and oscillatory regimes, which was obtained in the limit of nearly corotating 
cylinders. For values of $\operatorname{Pr} \geqslant 1.87$, Fig. 3 indicates that the upper branch corresponds to a secondary nonlinear transition to a stationary flow state. This is analogous to what is found for the rotating thermal convection, where "overstability" (an oscillatory onset of instability) is followed at higher Rayleigh numbers by a transition from oscillatory to nonoscillatory convective modes. ${ }^{14}$ The value of Prandtl number, which delimits the boundary between a stationary primary transition and an oscillatory primary transition, is a function of both $\eta$ and $N$. In summary, the main effect of a large Prandtl value is to induce a primary transition of the flow which is of Hopf type.

If we denote by $\mathrm{Re}_{2}$ the Reynolds number corresponding to the upper branch boundary in Fig. 3, we find that for $\operatorname{Pr}=50, \operatorname{Re}_{2} / \operatorname{Re}_{c 1}=0.644 / 0.411=1.567$, whereas the stability boundary between the $S$ and $S T$ regime in the laboratory experiments is such that $\left(\operatorname{Re}_{\mathrm{c} 2} / \mathrm{Re}_{c 1}\right)_{o b s}=0.625 / 0.409$ $=1.53$. The closeness of these relative Reynolds numbers suggests that the underlying cause for the transition between $S$ and $S T$ in BA's experiments is a temporal transition (from an oscillatory regime to a stationary one) in the axisymmetric part of the convective modes of stratified Couette flow. We shall return to this interpretation in Sec. IV and note moreover that the two branches which delimit the oscillatory and stationary regimes present the same asymptotic slopes for $\operatorname{Pr} \geqslant 16$. More specifically, the relative Reynolds number for $\operatorname{Pr}=16$ is $\operatorname{Re}_{2} / \operatorname{Re}_{c 1}=0.659 / 0.422=1.561$, and is therefore very close to the relative value found for $\operatorname{Pr}=50$. These numerical results suggest that the relative Reynolds number of the transition between the oscillatory and stationary regimes is a function of the Grashof number $G$ rather than the Rayleigh number $R=G P r$.

The numerical results for the large Pr part of Fig. 3 have been obtained by increasing the spatial resolution up to $256 \times 256$, until the critical value for $\Omega$ is found to vary by less than $0.2 \%$. The time and one-dimensional space resolutions which are required for establishing the upper branch of Fig. 3 vary roughly as $\operatorname{Pr}^{1 / 2}$ for the numerical code which is used, and thus correspond to an overall increase in cpu time as $\mathrm{Pr}^{3 / 2}$. This strong numerical constraint, together with the asymptotic tendency which is exhibited by Fig. 3, led us to opt for establishing the relative Reynolds number $\operatorname{Re} / \operatorname{Re}_{c 1}$ of the higher transitions while keeping a moderate value of $\operatorname{Pr}=16$ in the remainder of this work.

Finally, Fig. 3 has been established for purely axisymmetric simulations. In the case of three-dimensional simulations, the previous results are modified for Reynolds numbers such that $\mathrm{Re}_{\mathrm{Re}} \geqslant 1.18$, for which the primary axisymmetric flattened cells are slightly distorted by azimuthally dependent motions (see Sec. V).

\section{GROWTH OF AXIAL SCALES}

BA have identified three flow regimes $S, T$ and $C T$, with well-defined, regular density layers which are clearly visible in their shadowgraph visualizations. The corresponding layers heights for these regimes are respectively $n_{l}=h /(b-a) \approx \frac{1}{2}, 1$ and 2 , and their onset at $N=1.04 \mathrm{~s}^{-1}$ corresponds to a rotation speed of the inner cylinder of re- spectively $\quad \Omega_{c 1}^{o b s}=0.409 \mathrm{~s}^{-1}, \quad \Omega_{c 3}^{o b s}=0.90 \mathrm{~s}^{-1}, \quad$ and $\Omega_{c 4}^{o b s}=1.30 \mathrm{~s}^{-1}$. Flow regime $S T$, which is intermediate between the $S$ and $T$-regime, is such that density layer heights of both $n_{l} \approx \frac{1}{2}$ and $n_{l} \approx 1$ are present and its onset corresponds to $\Omega_{c 2}^{o b s}=0.625$.

\section{A. Three-dimensional simulations}

The initial conditions which are used in the threedimensional simulations consist in first attaining an equilibrium solution for the axisymmetric state and then to perturb this solution by random azimuthal noise. The simulation is then integrated until a flow regime takes over. All threedimensional simulations are performed for $\operatorname{Pr}=16$ according to the rationale which is presented at the end of Sec. III.

A clear increase of axial scale occurs for increasing Reynolds number Re. Figure 4 displays fields of azimuthal velocity deviation $q_{\theta}^{\prime}=q_{\theta}-\overline{q_{\theta}}$, in an axial-radial plane, for $\Omega=0.51,1.00,1.7$ respectively. These three simulations lie respectively within the $S, T$ and $C T$ regimes, and there is a clear increase in axial scales in the sequence of Fig. 4(a) to 4(c). The corresponding buoyancy fluctuation $\rho^{\prime}$ is shown in Fig. 5, with density layers heights which are respectively of $n_{l}=0.5,0.66,2.2$. Figure 6 displays the corresponding fields of the azimuthal vorticity component. We note the existence of a strong time variability and azimuthal dependence in all fields for the simulations which lie in the $S$ and $C T$ regimes, whereas the simulations of the $T$ regime display no timevariability nor azimuthal dependence of the flow.

On the other hand, it is interesting to contrast the $S$ and $T$ regimes to the $C T$ regime. In the first two cases, the density layer height $h$ is half the axial wavelength of the field of $q_{\theta}^{\prime}$, while in the $C T$ regime the density layer height is equal to the axial wavelength of the secondary motions. The doubling of the density layer height in the $C T$ regime is also clearly visible in the shadowgraphs of the laboratory experiments and corresponds to the intermittent disappearance of every second interface in density in both the laboratory experiments and in the numerical simulations. As commonly observed in the homogeneous fluid case, the vorticity field is concentrated at the level of the outflow jet between pairs of vortices, while the inflow jet is broader [Fig. 6(c)]. At large enough Reynolds number, a destabilization of the outflow jet occurs through shear instability, ${ }^{15,16}$ and this also occurs in the stratified fluid case. This process is very efficient at destroying the density front which is located at the same level. On the other hand, the more quiescent inflow jet is such that the density front at the same level remains very stable in time. We shall see in Sec. V that the $C T$ regime moreover displays a sizable azimuthal dependence at the outflow jet level.

\section{B. Axisymmetric stability boundaries}

The purpose of this section is to provide the evidence that for the stratified Couette-Taylor problem, the axial scale selection in $q_{\theta}^{\prime}$ is primarily determined by the axisymmetric part of the flow, while the azimuthally dependent component plays a lesser role. 


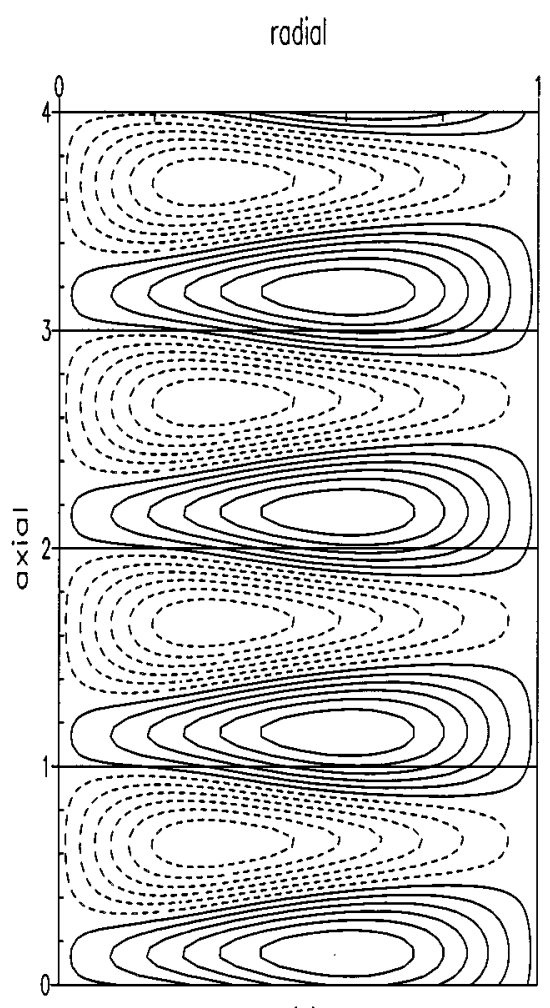

(a) radial

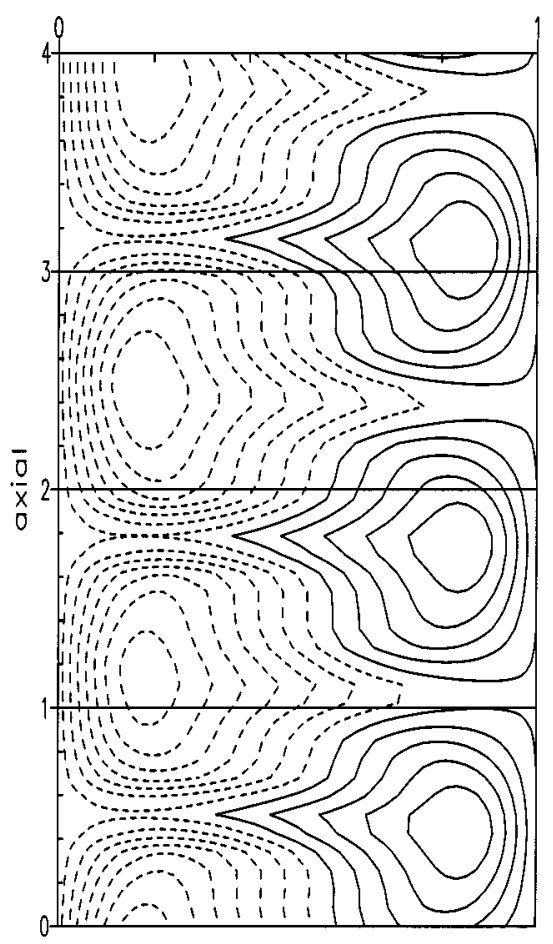

(b)

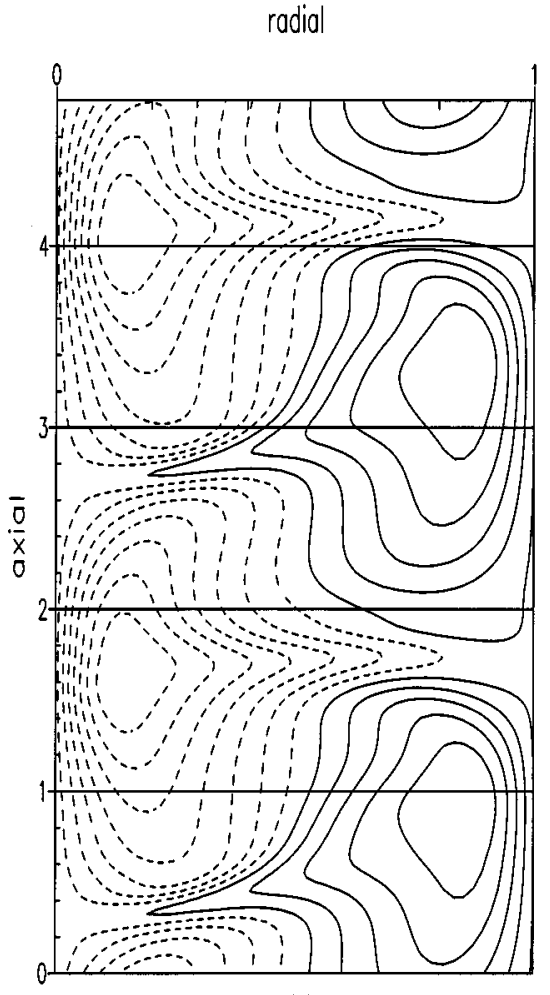

(c)

FIG. 4. Fields of azimuthal velocity deviations from purely azimuthal Couette flow $q_{\theta}^{\prime}$ in a $(r, z)$ plane for $\operatorname{Re} / \operatorname{Re}_{c 1}=1.20$ (a), 2.37 (b), 4.00 (c).

This interpretation was already implied by our remark at the end of Sec. III that the value of $\mathrm{Re} / \mathrm{Re}_{c 1}$ for the upper branch of the curve in Fig. 3 for $\operatorname{Pr}=16$, coincides rather closely with the transition value between the $S$ and $S T$ regimes which is observed in the laboratory experiments. This suggested that the $S$ to $S T$ transition is mainly induced by a temporal transition of the axisymmetric part of the flow. To pursue further this conjecture, we have determined the stability boundary for purely axisymmetric flows as a function of $L_{z \max }$ and $\Omega$, for the range of $\Omega$ spanning the regimes $S$ to $C T$. We denote by $L_{z \text { max }}$ the largest axial wavelength in the $q_{\theta}^{\prime}$ field, which is stable at finite amplitude at a given value of $\Omega$. Its value is determined by prescribing an initial disturbance, whose axial wavelength is identical to the domain of integration $L_{z}$. The numerical code is integrated forward in time, and if the finite-amplitude solution has an axial wavelength which is smaller than $L_{z}$, the axial height the domain of integration is changed to a smaller value. The procedure is repeated until the new flow state remains stable at an axial wavelength of $L_{z}$.

Figure 7 gives the resulting stability boundary in the case of $\operatorname{Pr}=16$. One can distinguish four regions in this diagram by their distinct temporal dependence.

For $0.42 \leqslant \Omega \leqslant 0.66$ and $1.0 \leqslant L_{z \max } / d \leqslant 1.6$, the flow is quasiperiodic, with an amplitude-modulation in time of all dynamical variables.

For $0.66 \leqslant \Omega \leqslant 0.96$, the upper part of the diagram is characterized by a chaotic behavior with a rich frequency spectrum for all fields. Moreover, several scales appear to take over at various instants in time. The lower part of the diagram corresponds to a stationary flow.

(iii) For $0.96 \leqslant \Omega \leqslant 1.53$, a stationarity of the structures is observed together with a strong variation of $L_{z \max }$ with $\Omega$.

(iv) For $\Omega \geqslant 1.53$, the flow is again oscillatory.

There is no jump in $L_{z \max }$ between the various regions of the diagram and the size of the toroidal vortices changes continuously with $\Omega$. The transition values of $R e / R_{c 1}$ between regions (i), (ii) and (iii) of Fig. 7, are respectively $1.56,2.27$ for the numerical simulations, while the laboratory experiments values are 1.53, 2.20 for the transitions between the $S, S T$ and $T$ regimes. The closeness of these relative Reynolds numbers supports our initial conjecture that at least the first two flow regimes have axial heights which are primarily set by the dynamics of the axially symmetric part of the flow. One can therefore identify the regions (i) and (ii) to BA's regimes $S$ and $S T$. On the other hand, the transition between regions (iii) and (iv) occurs at $\mathrm{Re} / \mathrm{Re}_{c 1}=3.62$, which is quite different from the experimentally measured value of 3.18 for the transition between $T$ and $C T$ regimes. We shall see in the next section that $C T$ is essentially determined by three-dimensional dynamics. In conclusion, only the low Re part of region (iii) can be identified with flow regime $T$.

\section{AZIMUTHAL DEPENDENCE}

The two-dimensional solutions of Fig. 7 have been perturbed by random three-dimensional disturbances in order to 
rodial

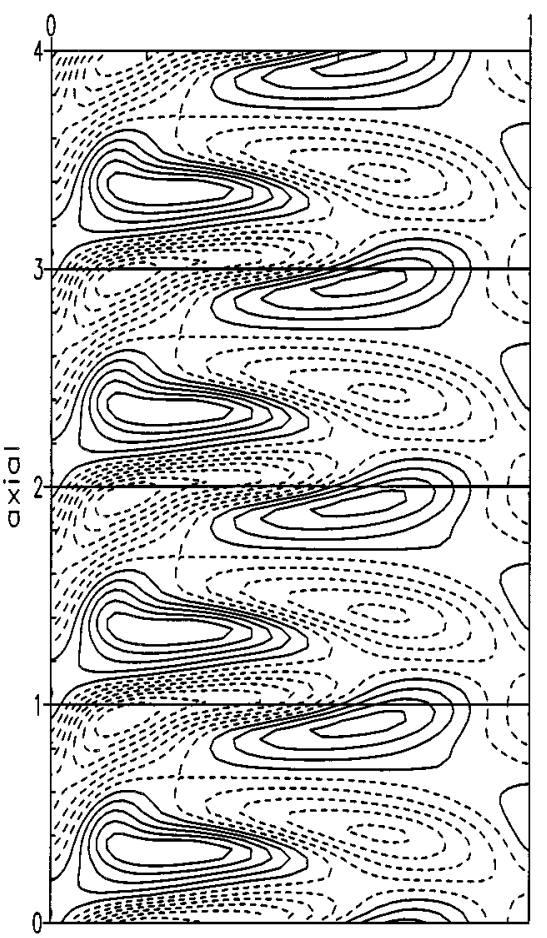

(a) radial

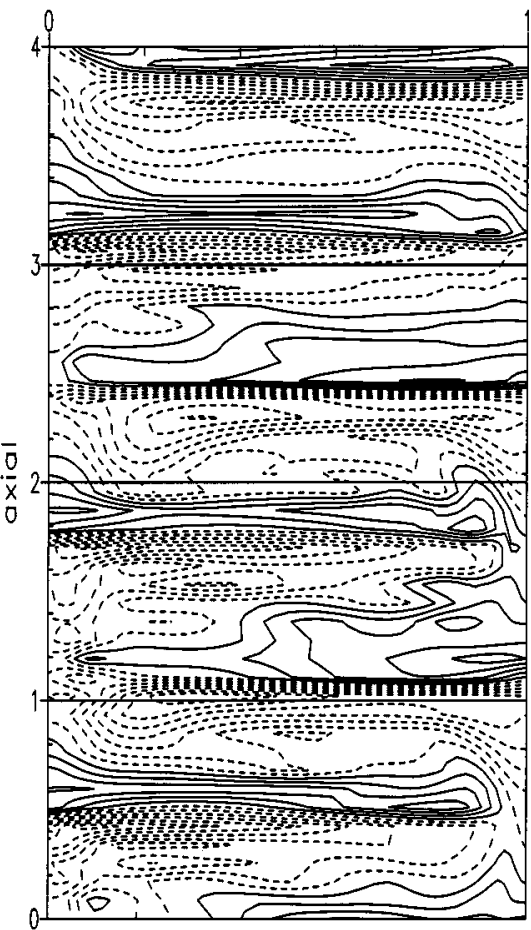

(b) rodiol

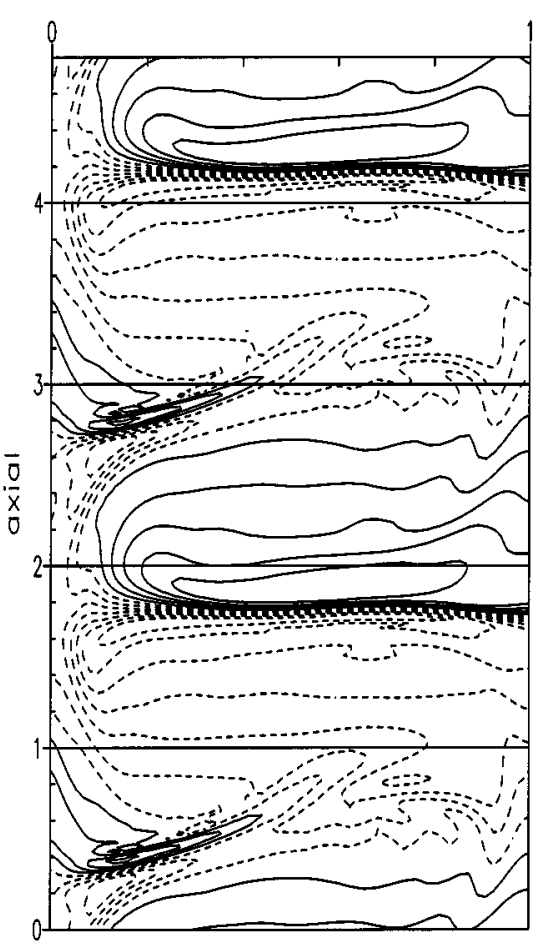

(c)

FIG. 5. Fields of buoyancy fluctuations $\rho^{\prime}$ corresponding to the same simulations than Fig. 4(a) to 4(c).

study the possibility of an azimuthal depence of the flow. The resulting stability limits to three-dimensional disturbances are indicated in Fig. 7 by vertical arrows along the abcissa. Finite-amplitude azimuthal perturbations are stable in both regions (i) and (ii) for $0.50 \leqslant \Omega \leqslant 0.96$, and also for $\Omega \geqslant 1.38$, for the high $\mathrm{Re}$ end of region (iii) and in region (iv). This last value of $\Omega$ is such that $\mathrm{Re} / \operatorname{Re}_{c 1}=1.38 / 0.422=3.26$ and thus lies within $2.5 \%$ of the experimental transition value between the $T$ and $C T$ regimes.

\section{A. $S$ regime}

The destabilization of the Taylor vortices by azimuthal modes is well documented for the homogeneous fluid case for the case of a fixed outer cylinder. ${ }^{12,15}$ This destabilization of the Taylor vortices occurs at higher Reynolds numbers than the destabilization of circular Couette flow and this is all the stronger as $\eta$ is large. Likewise, we observe that the stratified flattened cells in the $S$-regime are also destabilized by azimuthal modes for $\operatorname{Re} / \operatorname{Re}_{c} \geqslant 1.18$ at $N=1.04 \mathrm{~s}^{-1}$ for the case of $L_{z} / d=2$. This value moreover coincides with the result obtained at the same $\eta, L_{z} / d$, and $\nu$ for the homogeneous fluid case $(N=0)$. We observe an emergence of mode $m=3$, followed by $m=2$ and $m=1$ with increasing Re, and this is illustrated in Fig. 8 for $\operatorname{Re} / \operatorname{Re}_{c}=1.20,1.40$ and 1.54. Stratification inhibits the usual patterns which have been labelled as wavy Taylor vortices in the literature. Instead, BA report for $\mathrm{Re} / \mathrm{Re}_{c} \approx 1.3$ measurements of azimuthally dependent motions which have distinct periods of oscillation and preferred particle motions within a given horizontal plane.
For illustration, Fig. 9(a) displays the three-dimensional pattern which is observed at $\operatorname{Re} / \operatorname{Re}_{c 1}=1.40$ for the stratified case and for comparison, the wavy vortex pattern of the homogeneous fluid case at the same $\mathrm{Re} / \mathrm{Re}_{c 1}$ is shown in Fig. 9(b). The patterns clearly illustrate the preferred horizontal motions which are induced by stratification.

\section{B. $C T$ regime}

The other regime where a clear azimuthal dependence is observed in the numerical simulations is regime $C T$. Near onset of this regime, we show in Fig. 10 the azimuthal patterns in the field of $q_{\theta}^{\prime}$ which are observed for $\Omega=1.45$ and $L_{z}=2.3$. The corresponding radial-axial sections in $q_{\theta}^{\prime}, \rho^{\prime}$ and the azimuthal component of vorticity look very similar to Figs. 4(c), 5(c) and 6(c), respectively, and are not shown. There is a striking frontal wave pattern of mode $m=3$ in Fig. 10 , which is located right at the level of the outflow jet, while there is only a very weak azimuthal dependence at the inflow jet level. At this value of $\Omega=1.45$, the flow is still quasiperiodic, but it becomes quickly chaotic at larger values of $\Omega$.

The shear instability of the outflow jet is a threedimensional instability as in the homogeneous fluid case, ${ }^{15,16}$ and the pattern which is observed in Fig. 10 presents similar features to the so-called wavy outflow boundary (WOB) vortices which are commonly observed in the homogeneous case. We note, however, that the solution in Fig. 10 has an axial periodicity of $L_{z}$ and cannot therefore sustain an inter- 
radial

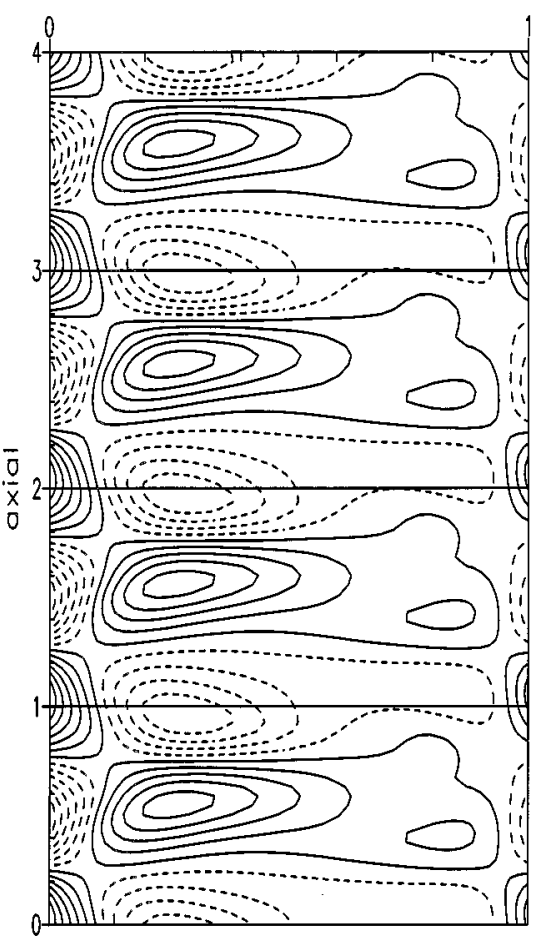

(a) radial

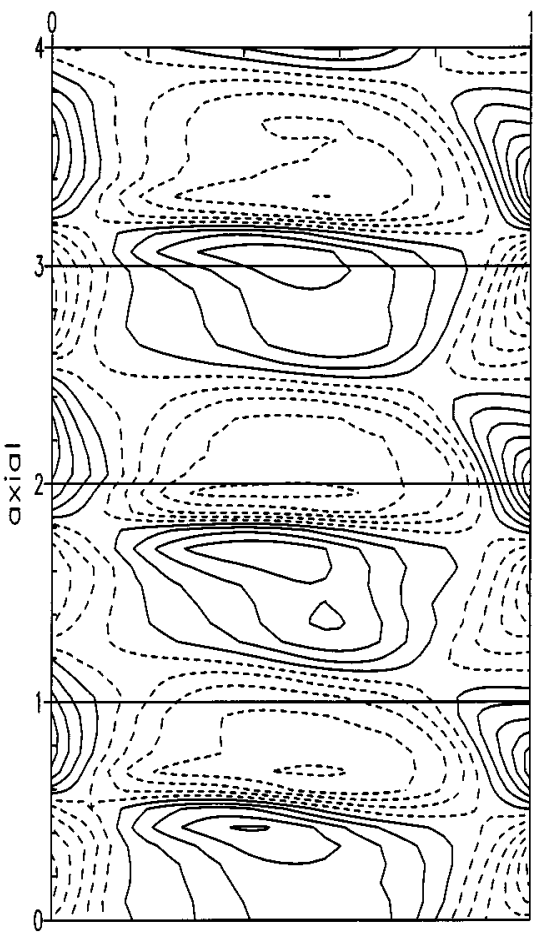

(b) radial

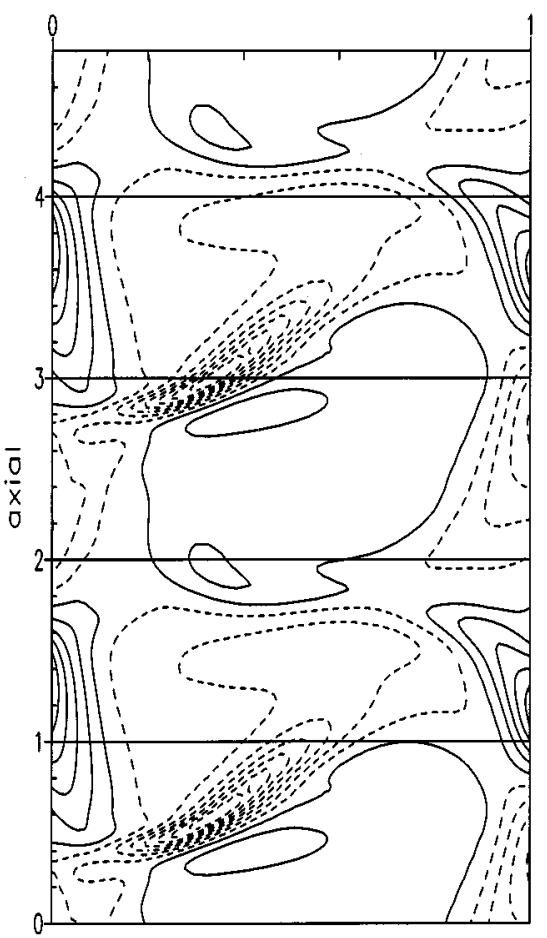

(c)

FIG. 6. Fields of azimuthal vorticity corresponding to the same simulations than Fig. 4(a) to 4(c).

action between axial scales $L_{z}$ and $2 L_{z}$ as discussed by Ioss. ${ }^{17}$ Finally, BA do not document the azimuthal patterns in regime $C T$, but we note that the azimuthal dependence at the outflow jet level is difficult to observe experimentally with only shadowgraph technique, since the density front at that level is suppressed intermittently.

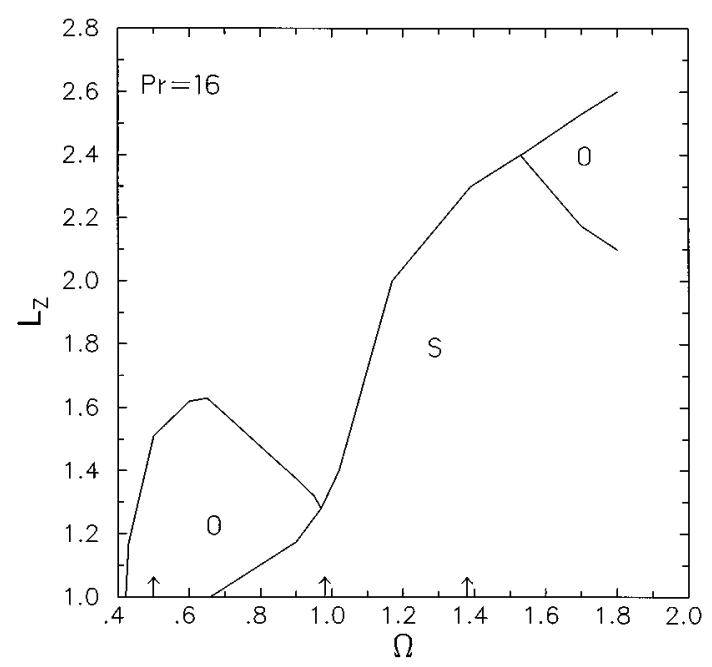

FIG. 7. Stability boundary of the tallest two-dimensional structures $L_{z \max } / d$ as a function of the inner cylinder rotation rate $\Omega$ for $\operatorname{Pr}=16$. The meaning of symbols $\mathrm{O}$ and $\mathrm{S}$ is the same than for Fig. 3. Vertical arrows delimit the stability boundary to three-dimensional disturbances (see section V).

\section{CONCLUSION}

The strongest influence of an axial stratification in density on the Couette-Taylor problem is the reduced aspect ratio of the secondary vortices. Stratification has a stabilizing influence which is quite analogous to the role of rotation in the thermal convection problem. We have shown in the Appendix how this stabilizing effect could be explained by the additive influence of the stratification parameter $R$ on the critical Taylor number of the flow.

A striking feature of the stratified problem is the growth of axial scales as a function of $\mathrm{Re} / \mathrm{Re}_{c}$. Our numerical simulations confirm that this observed growth of axial scale is mostly determined by the axisymmetric part of the flow, through its temporal behavior. The azimuthal dependence of the flow plays a lesser role in the determination of the axial scale. The simulations suggest a continuous growth of axial scale with increasing Reynolds number, which is at variance with the observations of the laboratory experiments, which protocole corresponded to an abrupt start of the inner cylinder rotation speed.

Despite looking rather different from the usual wavy vortices, stratified azimuthally dependent motions appear to follow closely the stability limit of the homogeneous problem at low Reynolds number. For increasing Reynolds number, azimuthal dependence disappears for the $T$ regime, while the $C T$ regime presents dynamical aspects which seem closely related to the WOB patterns of the homogeneous problem, albeit they do not involve an interaction between 


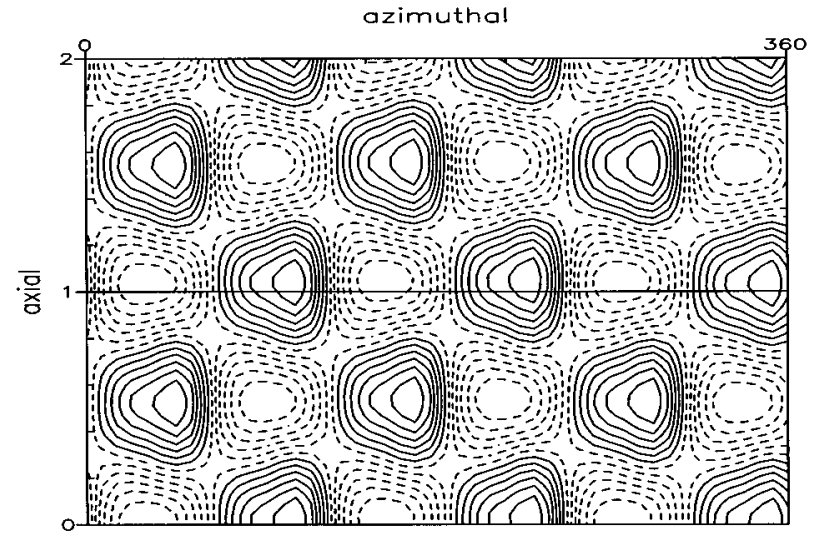

(a)

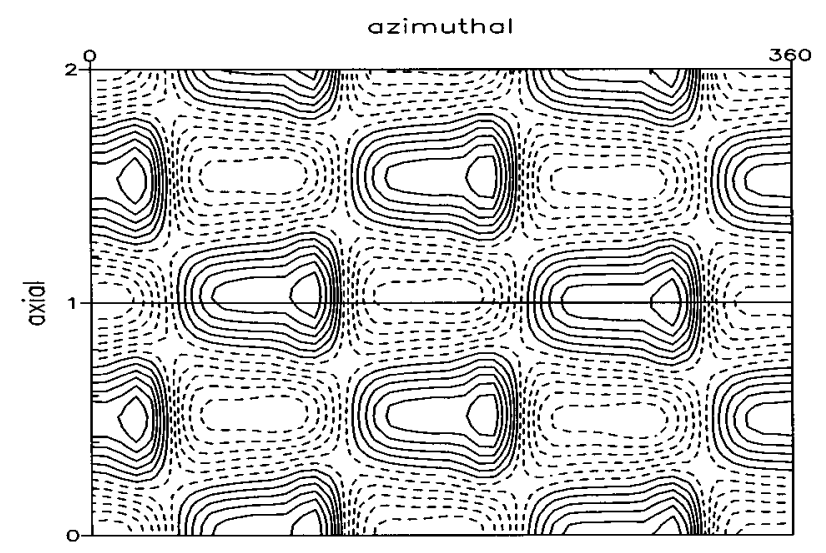

(b)

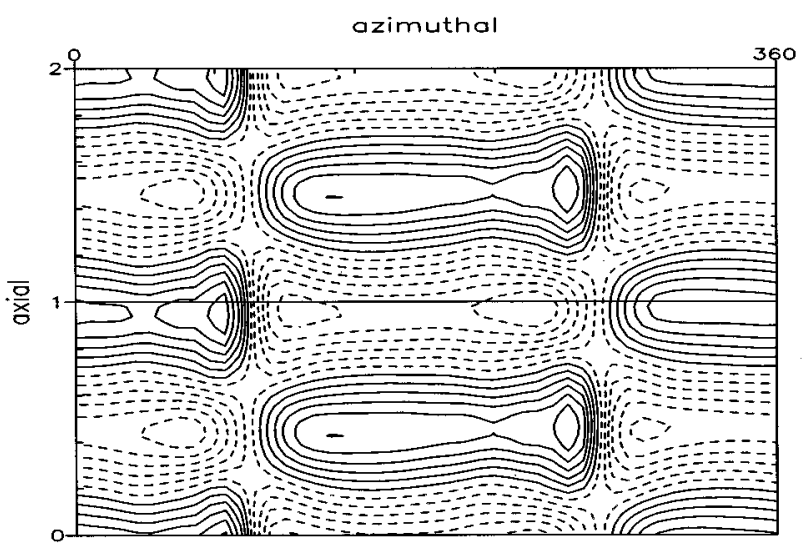

(c)

FIG. 8. Fields of $q_{\theta}^{\prime}$ in a $(\theta, z)$ plane for the first transitions in azimuthal wavenumber: $R e / \operatorname{Re}_{c}=1.20$ (a), 1.40 (b), and 1.54 (c). All cases lie in flow regime $S$.

axial scales $L_{z}$ and $2 L_{z}$. More work is needed to assess the extent of the analogy.

Finally, we note that a possible limitation of the present work is caused by the small range of nondimensional values of $L_{Z} / d$ which has been explored, thus ruling out the possibility of other solutions.

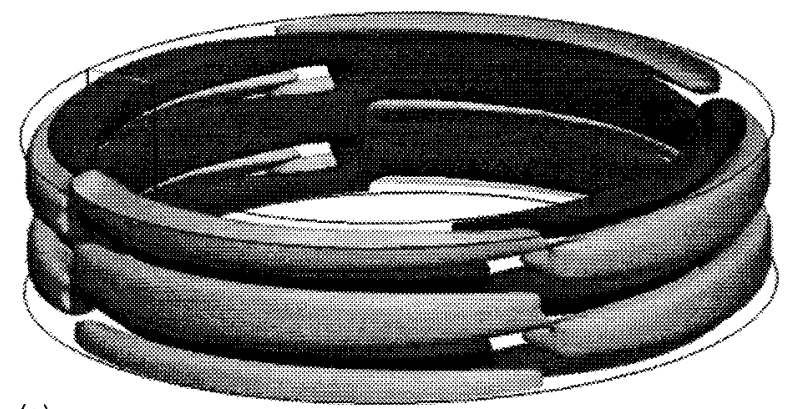

(a)

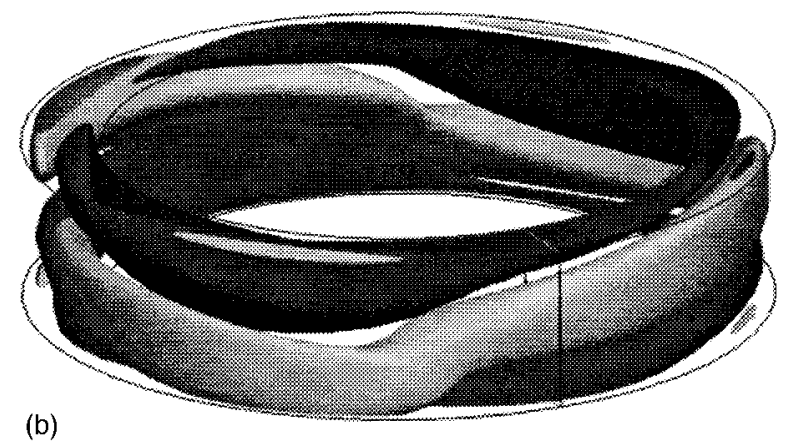

FIG. 9. Isosurfaces of $50 \%$ of the maximum of $q_{\theta}^{\prime}$ at $\mathrm{Re} / \mathrm{Re}_{c}=1.40$ for the stratified case (a) and for the homogeneous case (b).

\section{ACKNOWLEDGMENT}

Computing resources for this project were provided by Grant No. 950550 from IDRIS (Institut du développement et des ressources en informatique scientifique).

\section{APPENDIX: THE ANALOGY WITH ROTATING THERMAL CONVECTION PROBLEM}

We detail below the steps leading to an analytical solution for the primary onset of instability in the stratified Taylor-Couette problem, which is valid for a finite gap width, but corresponds to slightly different boundary conditions than in the laboratory experiments. The purpose of the analytical solution is to enable a validation of the stratified

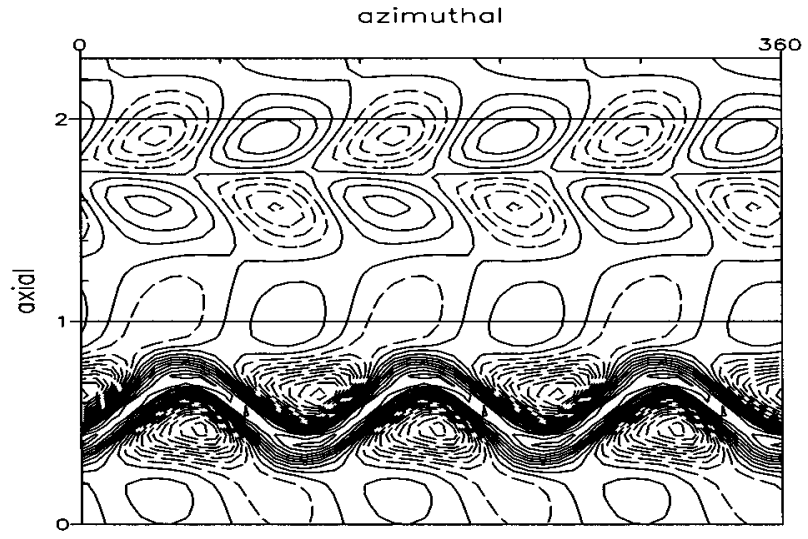

FIG. 10. Fields of $q_{\theta}^{\prime}$ in a $(\theta, z)$ plane for the $C T$ regime at $\Omega=1.45$ and $L_{z}=2.3 d$. 
version of Verzicco and Orlandi's code, ${ }^{2}$ and also to gain some physical insight on the stratified problem. The approach follows very closely the work of Thorpe,${ }^{10}$ in taking advantage of the dynamical analogy between the stratified Taylor-Couette (STC) problem and the rotating RayleighBénard (RRB) one, in the case of two-dimensional convective motions and for specific sets of boundary conditions, which are given below.

For axisymmetric motions and in the narrow gap approximation, the motions in STC can be described by the streamfunction $\Psi^{*}(x, z, t)$ of the convective modes, where $x$ and $z$ denote the radial and axial coordinates, the azimuthal velocity $v^{*}(x, z, t)$ and the density perturbation $\rho^{*}(x, z, t)$. [The radial and axial velocities are $u^{*}(x, z, t)=-\partial \Psi^{*} / \partial z$ and $w^{*}(x, z, t)=\partial \Psi^{*} / \partial x$.] Superscript $*$ is used to denote dimensional variables, but it has been omitted for coordinates in order to alleviate notations.

The linearized equations of motion are

$$
\begin{aligned}
& \left(\frac{\partial}{\partial t}-\nu \nabla^{2}\right) \nabla^{2} \Psi^{*}=\frac{g}{\rho_{0}} \frac{\rho^{*}}{\partial x}+2\left(\overline{\frac{q_{\theta}^{*}}{r}}\right) \frac{\partial v^{*}}{\partial z}, \\
& \left(\frac{\partial}{\partial t}-\nu \nabla^{2}\right) v^{*}=(-2 A) \frac{\partial \Psi^{*}}{\partial z}, \\
& \left(\frac{\partial}{\partial t}-\operatorname{Pr} \nu \nabla^{2}\right) \rho^{*}=-\frac{N^{2}}{g} \rho_{0} \frac{\partial \Psi^{*}}{\partial x},
\end{aligned}
$$

where coefficient $A$ corresponds to the circular Couette profile $q_{\theta}^{*}=A r+B / r$. We introduce the nondimensional quantities

$$
\begin{aligned}
& \Psi=\Psi^{*} /\left(\frac{g d^{3}}{\nu}\right), \\
& \rho=\rho^{*} / \rho_{0}, \\
& v z=-\frac{\partial v^{*}}{\partial z} /\left(\frac{g}{2 \Omega d}\right) .
\end{aligned}
$$

In order to reduce the STC problem to the RRB form, we need furthermore to use the "averaging approximation" 18 of replacing the angular velocity $\left(\overline{q_{\theta}^{*}} / r\right)$ by its average value in cylindrical coordinates [the averaging of angular velocity in cylindrical coordinates is equivalent to using a cartesian average of the Couette velocity profile]

$$
\begin{aligned}
\left\langle\overline{\frac{q_{\theta}^{*}}{r}}\right\rangle & =\frac{\Omega}{1-\eta^{2}}\left[-\eta^{2}+\frac{2 \eta^{2}}{1-\eta^{2}} \ln \left(\frac{1}{\eta}\right)\right], \\
& =\langle\mu\rangle \Omega .
\end{aligned}
$$

Assuming normal modes of the form

$$
\{\Psi, \rho, v z\}=\{\psi(x), \varrho(x), v(x)\} \exp (\text { inz }+\sigma t),
$$

with $D=d / d x$, we get

$$
\begin{aligned}
& \left(D^{2}-n^{2}-\sigma\right)\left(D^{2}-n^{2}\right) \psi=-D \rho+2\langle\mu\rangle v, \\
& \left(D^{2}-n^{2}-\sigma\right) v=-T n^{2} \psi, \\
& \left(D^{2}-n^{2}-P r \sigma\right) \varrho=R D \psi,
\end{aligned}
$$

where the Taylor and Rayleigh numbers are defined as
TABLE I. Results of direct numerical simulations at $\operatorname{Pr}=700$.

\begin{tabular}{lccc}
\hline \hline & Free-free & Experimental & Rigid-rigid \\
\hline$\Omega_{c 1}$ & 0.350 & 0.404 & 0.404 \\
$(2 h / d)$ & 1.12 & 1.03 & 1.03 \\
$\tau_{c}$ & 17.56 & 19.25 & 19.25 \\
\hline \hline
\end{tabular}

$$
\begin{aligned}
& T= \frac{4 A \Omega d^{4}}{\nu^{2}}, \quad R=\frac{\operatorname{Pr} N^{2} d^{4}}{\nu^{2}}, \\
& {\left[\left(D^{2}-n^{2}-\operatorname{Pr} \sigma\right)\left(D^{2}-n^{2}-\sigma\right)^{2}\left(D^{2}-n^{2}\right)\right.} \\
&\left.\quad+R\left(D^{2}-n^{2}-\sigma\right) D^{2}\right] \psi \\
& \quad=-\langle T\rangle n^{2}\left(D^{2}-n^{2}-\operatorname{Pr} \sigma\right) \psi,
\end{aligned}
$$

where we have denoted

$$
\langle T\rangle=\langle\mu\rangle T .
$$

Equation (A3) presents a very close similarity with equation (211) of Sec. 29 in Chandrasekhar, ${ }^{9}$ for the normal mode in vertical velocity in the RRB problem. The relevant Taylor number for the analogy is therefore not $T$ but rather $\langle T\rangle$.

The case for free-free, isothermal boundary conditions in $\mathrm{RRB}$ corresponds to the following set of conditions in STC ${ }^{10}$

$$
w_{x}=\rho_{x}=0, \quad u=v=0,
$$

while rigid-rigid, isothermal boundary conditions in RRB are analogous to

$$
w=\rho=0, \quad u=v=0 .
$$

However, for the experimental conditions of BA the appropriate set is intermediate

$$
w=\rho_{x}=0, \quad u=v=0,
$$

and there is no clear correspondence to physically meaningful boundary conditions in the RRB problem in that case.

The numerical results at onset of instability seem not too much altered quantitatively by the conditions at the boundaries. More precisely, the results of direct numerical simulations at $\operatorname{Pr}=700$ are listed in Table I.

At this large value of the Prandtl number, Table I reveals that there is no discernable change in the parameters at onset of instability between the experimental boundary conditions and the rigid-rigid set of conditions (A5). Furthermore, when going from the experimental set of boundary conditions to the free-free set of conditions (A4), numerical results are modified by $8 \%$ to $16 \%$, depending on which dynamical index is considered. This relative insensitivity of the results suggests to take advantage of the straightforward analysis in RRB for free-free boundary conditions, and to study the solution of equation (A3) with the set of boundary conditions (A4), in order to gain some physical insight on the problem. The corresponding solutions are of the form $\psi=\psi_{0} \sin (\pi x)$, and for an oscillatory solution such that $\sigma=\sqrt{-1} \sigma_{1}$, one finds

$$
\sigma_{1}^{2}=\frac{1}{\operatorname{Pr}^{2}}\left[\frac{R}{(1+\chi)} \frac{\operatorname{Pr}-1}{2}-\pi^{4}(1+\chi)^{2}\right], \quad \chi=\frac{n^{2}}{\pi^{2}}
$$


and

$$
\langle T\rangle=\frac{1}{\chi}\left[\pi^{4}(1+\chi)^{3}\left(\frac{\operatorname{Pr}+1}{\operatorname{Pr}}\right)^{2}+\frac{R}{2 \operatorname{Pr}^{2}}(\operatorname{Pr}+1)\right] .
$$

(The only difference between our solutions and those of Thorpe $^{10}$ is the introduction of $\langle T\rangle$ instead of $T$. This leads, however, to large quantitative changes in the solutions.) The right-hand side of (A8) shows that $\langle T\rangle$ is an increasing function of $R$, and thus proves the stabilizing influence of both the stratification and of large values of the Prandtl number, through their combined influence on the Rayleigh parameter $R$. One can compute from (A8) the marginal conditions for instability at $d\langle T\rangle / d \chi=0$. The values of $\operatorname{Re}_{c 1},(h / d)$, and $\tau_{c}$ which are thus obtained (respectively $0.348 \mathrm{~s}^{-1}, 1.12$, $17.48 \mathrm{~s}$ ) agree within $0.6 \%$ with the direct numerical simulations of STC with free-free boundary conditions which are listed in Table I.

Furthermore, one can derive the asymptoptic expression at large values of $[R /(\operatorname{Pr}+1)]$, leading to a $N^{2 / 3}$ law for the inner cylinder rotation speed $\Omega_{c}$ and to a $N^{1 / 3}$ law for the density layers height $(h / d)$. However, we have checked through numerical simulations that such an asymptotic regime should be relevant for values of buoyancy frequency $N$ which are at least ten times larger than the range of experimental values which are used in BA.

At large values of the Prandtl number $(\operatorname{Pr} \gg 1)$, the oscillation period at onset of instability can be obtained from (A7):

$$
\tau_{c}=\sqrt{2(1+\chi)} \frac{2 \pi}{N} .
$$

The oscillation period is thus strongly dependent on the "natural" oscillation period of the stratified fluid $2 \pi / N$. Moreover this expression is also found to be a good approximation for other types of boundary conditions than (A4), since it yields $\tau=18.66 \mathrm{~s}$ [for the value of $h / d=0.515$ which is observed at onset of instability], i.e., it differs by only $3 \%$ from the numerical results which yield $\tau=19.25 \mathrm{~s}$.

We have so far only considered the case of an oscillatory primary transition. For a value of $\operatorname{Pr}=1$, the primary transition is stationary and using the same approach than above for $\sigma=0$, we find that the analytical results agree more accurately with the direct numerical solutions of STC with the set of boundary conditions (A4), if we replace formula (A2) by the original formula of Taylor ${ }^{19,18}$ for the influence of the finite gap width

$$
\langle T\rangle=\frac{T}{2[1+0.652(1-\eta)]} .
$$

The latter expression differs from (A2) by $4.8 \%$ at $\eta=0.775$.
In summary, for the case of a primary transition which is of Hopf type and at large Prandtl number, direct numerical simulations show that the onset of instability is rather insensitive to the nature of the boundary conditions. An analytical approach, which uses an averaging approximation based upon the mean angular velocity (A2) in cylindrical coordinates, leads to a solution which agrees within $0.6 \%$ with the numerical simulations for the corresponding boundary conditions. Such solution is analogous to the rotating thermal convection solution for free-free boundary conditions. We do not have a formal rationale why the averaging approximation works better for the case of an oscillatory primary transition than for a stationary one.

${ }^{1}$ B. M. Boubnov, E. B. Gledzer, and E. J. Hopfinger, "Stratified circular Couette flow: Instability and flow regimes,' J. Fluid Mech. 292, 333 (1995).

${ }^{2}$ R. Verzicco and P. Orlandi, "A finite-difference scheme for threedimensional incompressible flows in cylindrical coordinates,' J. Comput. Phys. 123, 402 (1996).

${ }^{3}$ B. M. Boubnov, E. B. Gledzer, and E. J. Hopfinger, "Corrigendum of 'Stratified circular Couette flow: instability and flow regimes',' J. Fluid Mech. 292, 333 (1996).

${ }^{4}$ R. C. Di Prima and H. L. Swinney, "Instabilities and transition in flow between concentric rotating cylinders," in Hydrodynamic Instabilities and the Transition to Turbulence, edited by H. L. Swinney and J. P. Gollub (Springer, New York, 1981), p. 139.

${ }^{5}$ R. Tagg, "The Couette-Taylor problem,'” NonLin. Sci. Today 4, 1 (1994).

${ }^{6} \mathrm{~T}$. Dunkerton, "On the inertial stability of the equatorial middle atmosphere,'’ J. Atmos. Sci. 38, 2354 (1981).

${ }^{7}$ D. Stevens, “On symmetric stability and instability of zonal mean flows near the equator,', J. Atmos. Sci. 40, 882 (1983).

${ }^{8}$ B. L. Hua, D. W. Moore, and S. Le Gentil, "'Inertial nonlinear equilibration of equatorial flows,', J. Fluid Mech. (in press).

${ }^{9}$ C. Chandrasekhar, "Hydrodynamic and hydromagnetic stability.', (Dover, New York, 1961), p. 652.

${ }^{10}$ S. A. Thorpe, "The stability of stratified Couette flow,', in Notes on 1966 Summer Geophys. Fluid Dynamics (Woods Hole Oceanographic Inst., Woods Hole, MA, 1966), p. 80.

${ }^{11} \mathrm{G}$. Veronis, "The analogy between rotating and stratified fluids,' Annu. Rev. Fluid Mech. 2, 37 (1970).

${ }^{12}$ A. C. Jones, "Nonlinear Taylor vortices and their stability,' J. Fluid Mech. 102, 240 (1981).

${ }^{13}$ O. M. Rayleigh, "On the dynamics of revolving fluids,' Proc. R. Soc. Serv. A 93, 148 (1916).

${ }^{14}$ N. O. Weiss, "Convection in the presence of constraints,", Philos. Trans. R. Soc. Lond. Ser. A 256, 99 (1964).

${ }^{15}$ A. C. Jones, "The transition to wavy Taylor vortices," J. Fluid Mech. 157, 136 (1985)

${ }^{16}$ K. T. Coughlin and P. S. Marcus, "Modulated waves in Taylor-Couette flow. Part 2. Numerical simulation,' J. Fluid Mech. 234, 19 (1992).

${ }^{17} \mathrm{G}$. Ioss, "Secondary bifurcations of Taylor vortices into wavy inflow or outflow boundaries,' J. Fluid Mech. 173, 273 (1986).

${ }^{18}$ P. G. Drazin and W. H. Reid, Hydrodynamic Stability (Cambridge U. P., Cambridge, 1981), p. 527.

${ }^{19}$ G. I. Taylor, "Stability of a viscous liquid contained between two rotating cylinders,' Philos. Trans. Sec. A 223, 289 (1923). 